\title{
Differential GPS Integrity Monitor
}

\author{
Joseph W. Spalding \& Lee A. Luft \\ USCG R\&D Center \\ 1082 Shennecossett Road \\ Groton, CT 06340-6096
}

ABSTRACT

Government provided DGPS service is currently planned for all coastal waters of the US. The Harbor/Harbor Approach (HHA) requirement from the Federal Radionavigation Plan [1] will be met by the Coast Guard's DGPS senvice. Commercial shipping will rely heavily on the DGPS service for maneuvering in harbors and constrained waterways. Therefore, the Coast Guard has developed a requirement for real time integrity monitoring of the DGPS service. In order to fulfill this requirement, the United States Coast Guard R\&D Center has been conducting research regarding integrity monitoring of Differential GPS (DGPS). The research is currently focused on the development of a prototype integrity monitoring system. This paper describes this DGPS integrity monitor and the specific DGPS quantities monitored. These include the radiobeacon signal, the quality of the RTCM SC-104 message stream and the accuracy of the positioning performance using the DGPS corrections. Algorithms are described for all quantities measured. Details of the software design and implementation, including the use of standard RTCM SC-104 [3] and NMEA-0183 [4] data interfaces are included.

\section{INTRODUCTION}

Since 1991 the United States Coast Guard R\&D Center has been conducting research regarding integrity monitoring of Differential GPS (DGPS). The research is currently focused on the development of prototype integrity monitoring software named Reference Station Monitor (RSM). RSM is designed to detect and raise alarms based on anomalous conditions in the Coast Guard DGPS service. This paper documents the history of the RSM effort including key decisions in selecting design methods and the programming language. The RSM consists of external equipment and internal software. The external equipment requirements consist of a DGPS receiver, MSK beacon receiver, control station, and a data logger. The internal software structure is presented component by component. Individual algorithm discussions and functional descriptions are covered under the component where they reside. The RTCM SC-104 decoding process and the GPS receiver interface are described. User displays are presented and there is a full discussion of the RTCM
SC-104 Reference Station Integrity Monitor (RSIM) [2] standard implementation complete with alarms for the specific DGPS quantities monitored.

\section{BACKGROUND}

In 1991 the requirement for a Differential Global Positioning System (DGPS) integrity monitor was identified by the USCG DGPS implementation team. At that time the requirements for an integrity monitor were undefined. As a member of the implementation team, the Advanced GPS project at the R\&D Center endeavored to create a prototype of this integrity monitor. Researching the requirements and developing this part of the DGPS service was a natural extension of previous R\&D DGPS activities.

RSM is being developed in the Ada programming language because maintainability and portability were of great concern during language selection. Maintainability features are important because large portions of the source code are being developed by contract programmers while other portions are developed by government programmers. When complete, the source code will be handed off to the USCG Electronics Engineering Center for maintenance by a similar combination of government and contract programmers. Portability was a key issue due the rapid pace of change in computer hardware combined with the expected life of the software.

The design and development of the RSM software employed the 'Evolutionary Software Development Process' as outlined in the Software Management Guide, published by the Department of Defense Software Technology Support Center (STSC) as document MAC010/STSC. The 'evolutionary' approach breaks down the software development effort intomanageable sizes or phases, and provides intermediate product releases that are used for the final system. Utilizing the 'evolutionary' approach, RSM was designed and developed with modular or compartmentalized components. When put together, these components make up the complete RSM. This allows for easy design changes, and provides the ability to generate variants of RSM by simply choosing which components are needed for a given application. Each of the components are reusable, and can be configured

\section{5}

U.S. Government work not protected by U.S. Copyright. 
to support other software applications.

Another main philosophy in the development was the strict adherence to existing industry standards. Standards used include: RTCM SC-104 Recommended Standards For Differential Navstar/GPS Service (version 2.0) [3], RTCM SC-104 Recommendations for Reference Stations and Integrity Monitors (version 1.0 draft) [2], NMEA-0183 Standard For Interfacing Marine Electronics (version 2.0) [4].

The RSM software is developed and tested on a Hewlett Packard series 9000 model 380 running the HP-UX 8.00 (UNIX) operating system. This platform provides a multiple $X$-Window environment suitable for software development. The RSM software source code, once fully tested, is moved electronically to a Compaq model 486/50L computer running the MS-DOS 5.0 operating system. The software is re-compiled under MS-DOS and tested. Unique $1 / O$ resources are handled locally on each platform. The actual port of the software takes approximately two to three days by a cognizant programmer. The DOS executable is a full 32 bit application, and will not operate on anything less than an $803865 X$ processor.

\section{EXTERNAL EQUIPMENT}

This section identifies and describes the type of external equipment that is supported by the RSM program (figure 1). Any compatibility issues, limitations, or important selection criteria are brought to the readers attention. Though a wide variety of equipment may be connected to RSM, only a small cross-section of the available equipment provides enough timely information to properly support the integrity functions of the RSM.

\section{DGPS Receiver}

The Global Positioning System (GPS) or Differential GPS (DGPS) receiver is commonly referred to as User Equipment (UE). The UE provides the RSM with navigation solutions and related information. This includes satellite Signal to Noise (SNR) ratios, azimuth and elevation for each tracked satellite, the satellite constellation used to produce the navigation solution, the Horizontal, Position, and Altitude or Vertical Dilution of Precision (HDOP, PDOP, VDOP) values, and satellite almanacs.

The logical interface to RSM consists of the format in which data and measurements are moved from the UE to the RSM. The format supported by RSM for UE output is the National Marine Electronics Association (NMEA 0183) Standard for Interfacing Marine Electronics, version 2.0 [4]. The only UE tested to date

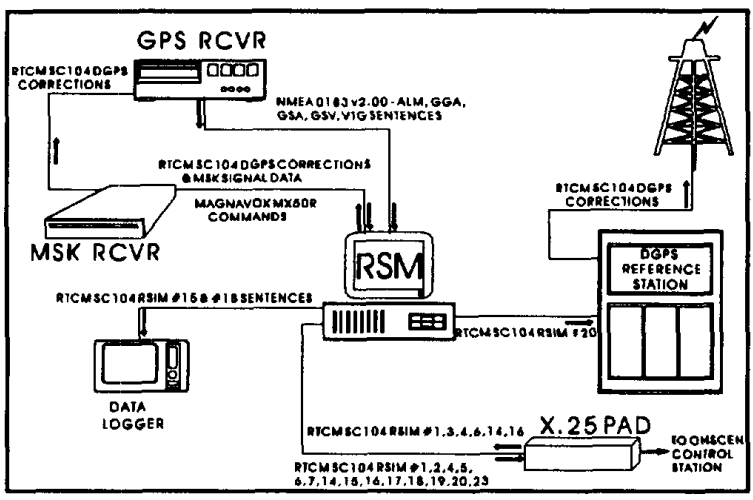

Figure 1 External Equipment

has been the Ashtech Ranger GPS receiver. RSM requires five different data sentence formats, as defined in the NMEA 0183 v2.0 standard. They are the GGA (geographic position), GSV (satellites in view), GSA (satellites in navigation solution), VTG (velocity), and ALM (GPS satellite almanac) formats. The combination of these specific messages provide RSM with enough information to perform the integrity monitoring of the GPS/DGPS navigation solution.

Selection of a specific User Equipment for operation with RSM depends on the application of RSM. In an integrity monitoring application, RSM requires that the UE meet all the criteria below. Any other application of $R S M$ requires that the UE have at least the first three capabilities.

1. The UE must have a RS-232 serial interface for data output, or there must be a method to convert its interface to RS-232.

2. The UE must be able to output data at some continuous rate, and it must be in the NMEA 0183 v2.0 standard format [4].

3. The UE must be able to generate and send the GGA, GSA, GSV, and VTG message formats as specified within the NMEA 0183 v2.0 standard [4].

4. The UE should have an additional port or method to receive differential corrections.

5. The UE should be an all-in-view receiver for best results.

6. The UE should be able to generate and send the ALM message format as specified within the NMEA 0183 v2.0 standard [4].

\section{MSK Radio Beacon Receiver}

An important aspect of integrity monitoring with RSM is the ability to monitor both the content and signal quality of the differential broadcast. A MSK Radio Beacon receiver is used to receive the Radio Beacon differential 
broadcast. The MSK receiver takes the DGPS message data from the Minimum Shift Keying (MSK) modulated signal, and places it into the American Standard Code for Information Interchange (ASCII) format. Within the ASCII format, the data is structured to meet the "6 of 8" rule, from chapter five of RTCM SC104 [3]. This formatted data is then passed from the MSK receiver to the RSM program.

The logical interface between the MSK receiver and the RSM consists of the differential message data formatted as specified in the RTCM SC-104 $\sqrt{2.0}$ standard [3]. The RSM program can receive and decode the differential messages only if they are formatted in this manner. The RSM can communicate with the MSK receiver, for purposes of setup (radio beacon frequency selection and bit rate), and to request current setup, signal strength, and signal to noise measurements. A format for receiver control and measurement queries was developed by Magnavox Advanced Products and Systems Company of Torrance California for their MX50R Beacon Receiver product. This command structure format is used by RSM, and is emulated by several other MSK radio beacon receiver manufacturers.

Selection of a specific MSK radio beacon receiver for operation with RSM depends on the application of RSM. In a DGPS integrity monitoring application, RSM requires that the MSK receiver meet all the criteria below. Any other application of RSM requires a MSK receiver to have at least the first three capabilities.

1. The MSK receiver must have a RS-232 serial interface for data output, or there must be a method to convert its interface to RS-232.

2. The MSK receiver must be able to output data in adherence to the RTCM SC-104 version 2.0 [3].

3. The MSK receiver must be able to output signal strength and signal to noise measurements in the format specified by the Magnavox MX50R manual.

4. The MSK receiver should be able to be remotely controllable via Magnavox's MX50R format for setup and control.

5. The RTCM output of the MSK receiver should also be connected to the UE's differential input port.

\section{Control Station}

A major component in the U.S. Coast Guard's DGPS Service architecture is the Control Station (CS). The Coast Guard implementation of the DGPS service calls for two control stations, one on each coast. Although each DGPS Broadcast site is designed to operate autonomously, the CS monitors their performance, and has full remote control capability if needed. The CS is connected to RSM via an X.25 Network using a Packet Assembler/Disassembler (PAD). The logical connection consists of the format and content of the information that moves between the CS and RSM. The information must adhere to the RTCM SC-104 recommended standards for differential navstar GPS Reference Stations and Integrity Monitors (RSIM) [2]. The RSIM document defines the form, content, and application of twenty three different messages. The RSM uses a subset of these messages consisting of \#1, $\# 2$, \#5, \#6, \#7, \#14, \#15, \#16, \#17, \#18, \#19, and \#23. This combination of messages provides query, control, alarm and data reporting capabilities.

\section{DGPS Reference Station}

Differential GPS corrections and other RTCM SC-104 messages are generated by the Reference Station (RS). The connection between the RS and RSM is used by the RS to determine if it is being monitored by the RSM. The RS indicates if it is monitored or unmonitored by setting two data bits within the header word of every RTCM message generated for broadcast. The logical connection is composed of the RSIM $\# 20$ message. This message is sent frequently to the RS by RSM, and includes indications of normal or abnormal operation.

\section{Data Logger}

The RSM program can send RSIM \#15 and \#18 messages to any available serial ports. The CS can configure the RSM to send either a single instance or a continuous output of the RSIM \#15 and \#18 data messages. Whenever these are sent to the CS, they are also echoed to the data logging port selected. The logical connection consists of the RSIM \#15 and \#18 messages. How often they are sent to the logging device is determined by the CS. The RSM program does not care whether a logging device is connected or not.

\section{INTERNAL STRUCTURE}

The Reference Station Monitor program consists of six major components. These software components are the Data Handler, RTCM Decode, User Equipment, Engineering Display, Watchstander Display, and RSIM Manager (figure 2). Each of the six components are described in the following subsections. In addition to these six, a section titled 'Alerts' describes several alarm-like events, and a section describing the various methods of controlling operation of RSM are included. 


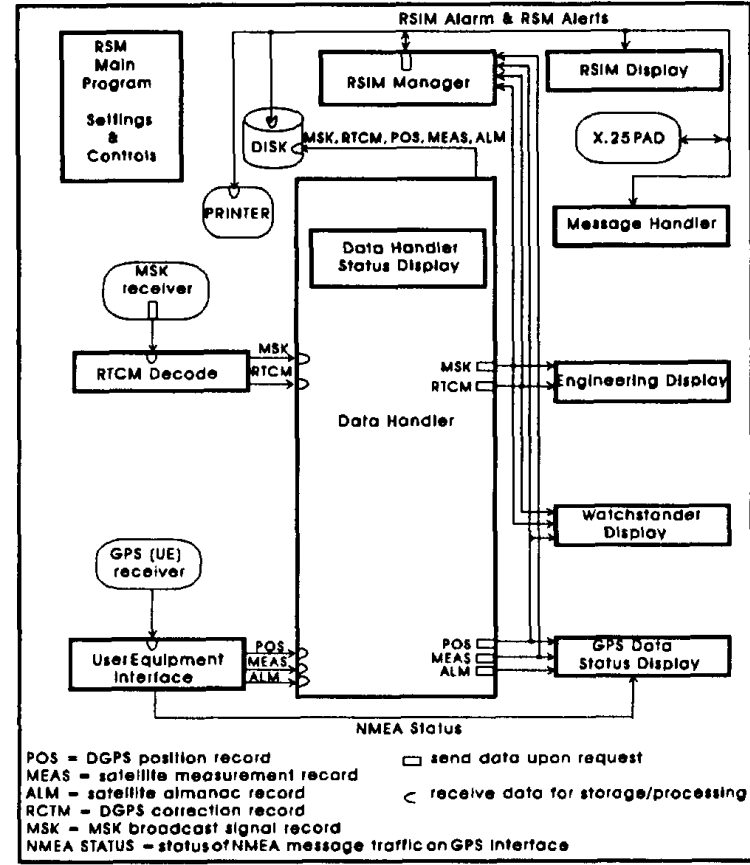

Figure 2 Internal Structure

\section{Data Handler}

The Data Handler acts as the central data repository for RSM. This is the core facility within RSM, and consists of a single generic data buffer with an internal Ada task to manage buffering, storage, retrieval, and data exporting of all data. The generic buffer is instantiated for each of the five main data records that RSM manages. This design facilitates the addition of new data types or controls in a clear and maintainable manner.

The Data Handler accepts binary data records from the RTCM Decode package \& User Equipment package. RTCM, MSK signal, position,measurement and almanac are the names of these data records. In this document all references to these particular data blocks are italicized. This will help differentiate the data blocks for the reader of this document as these names have common usage in GPS and DGPS. Upon request the Data Handler will provide the most recent data records for use in other parts of the program. The Data Handler maintains a dynamic and controllable buffer in RAM for each type data record it receives. Data buffering and storage controls are provided for each type of data record. These controls allow the user to set the size of each buffer in units of time (seconds), set how often the data should be written from the buffer to disk, set a sample interval in seconds to control the number of records stored (the time of the data record is checked against this interval), and set a receiver identifier for use in the data file name. Once data has been passed in to the Data Handler, it can not be modified in any way. This preserves the integrity of the information. When another section of the program desires data, it is given a copy of the data requested, and then allowed to perform any alteration as needed on that copy.

\section{RTCM Decode}

The Reference Station Monitor can receive and decode RTCM SC-104 formatted DGPS corrections. RTCM message formats recognized by RSM include the following types: $1,2,3,5,6,9$, and 16 . The source of the RTCM messages is inconsequential, so long as they are received on one of the PC's serial ports. In addition to RTCM messages, Signal Strength (SS) and Signal to Noise (SNR) measurements of the radio beacon broadcast signal are also decoded. The SS and SNR measurement formats must conform to the Magnavox data format used in their MX50R MSK RadioBeacon Receiver product. This information is received as embedded data within the RTCM message stream.

Once data is received, the RTCM decode begins by checking the validity of the data byte. RSM uses the 6 of 8 rule as found in chapter five of the RTCM SC104 document [3]. Based on that rule, only bytes in the decimal range of 64 to 127 are considered valid RTCM data. If the received byte is not within that range, then it is filtered out for possible SS/SNR measurement decoding. The decode process continues by looking for the RTCM preamble and valid parity bits. Once the preamble has been identified, and the parity check has passed, the rest of the RTCM message is put together and checked for parity and proper length. The process continues by converting this binary data into actual values, depending on the message type as indicated in the header words. At this point, an RTCM data record is formed with the decoded values and passed to the Data Handler for storage and distribution within the RSM program. The decode process continues in this cycle as long as data is received.

When a complete set of SS/SNR data bytes have been received and decoded, the measurements are placed into a MSK signal data record and sent to the Data Handler for storage and distribution within the RSM program.

There are two other major components of the RTCM decode process. They are the Message Error Rate (MER) calculations, and the actual state of the decode process. The MER calculation is based on the number of bad bits (data that did not pass the parity check) 
versus the number of good bits (data that was decoded properly). If any part of a RTCM message is bad, then the entire message (all the bits) are perceived as bad. The MER calculation makes use of a sliding window of a certain size. If the window was set to 1 minute in length, then it would hold 6000 data bits (100 bps broadcast). Each time new data is received, it is placed in the sliding window, causing the oldest data to be bumped out (assuming the window was full). The actual MER calculation is updated when a new RTCM message or SSISNR measurement data is received, or at twice the SS/SNR polling rate if no SS/SNR data is received (i.e. a receiver that does not support the Magnavox MX50R SS/SNR data format). The MER value is placed in the MSK signal data record and sent to the Data Handler for storage and distribution within the RSM program. There are five different states that can occur during the RTCM decode process. Any change in state is considered significant, causing an alert to be generated. The states and their meaning are summarized here:

RCVR_NOT_CONFIGURED - This state indicates that the MSKK Beacon receiver has not been configured. Detection of this state is dependant on values of SS and SNR retumed by the beacon receiver as specified in the Magnavox MX50R Beacon receiver manual.

NO_MSK_SIGNAL - This indicates that the MSK Beacon receiver is unable to track or detect any MSK modulated signal at the set frequency. Detection of this state is dependant on values of SS and SNR returned by the beacon receiver as specified in the Magnavox MX50R Beacon receiver manual.

WAITING FOR DATA - This state occurs when there is no data being received on the interface selected for RTCM data.

TRYING TO LOCK - This indicates that the RTCM decode process is receiving data, but is searching for a RTCM message preamble.

PREAMBLE_DETECTED - This state identifies the point in the decode process when a RTCM preamble has been located, indicating that the beginning of a RTCM message has been found.

LOCKED_ON_RTCM_DATA - This is the ideal or normal state. This indicates that the decode process is locked on to the synchronous RTCM data stream, and continually decoding valid RTCM messages.

The above decode process states are placed in the MSK signal data records for storage and propagation within the RSM program. Whenever a change in state occurs, a new MSK signal record is generated, with current SS/SNR and MER values. The current state of the RTCM decode process can be readily found on several different displays.

\section{User Equipment}

The term User Equipment (UE) corresponds to a DGPS receiver used to obtain navigation solutions and satellite signal and constellation data. This package generates three types of data records, position data, satellite measurement data, and satellite almanac data. The particular NMEA 0183 V2.0 [4] data messages required are the GGA, GSA, GSV, VTG, and ALM. The UE must be setup to continually output the NMEA messages listed above, except for the ALM message.

The ALM or GPS almanac message is too large for continuous output. When the user desires a new almanac the message should be enabled and then disabled once the RSM indicates reception.

The UE Package is comprised of four separate packages. The first package contains one Ada Task for reading data from the receiver, and passes that data to a second package. This package breaks each NMEA 0183 v2.0 [4] data sentence into its component fields and determines what type of method (UE/GPS receiver dependent) should be used to decode the fields. Once the decoding method has been identified, the component fields are sent to the package(s) that support that method. The last package places the decoded and validated data into predefined data records (position, measurement, almanac), and sends them to the Data Handlersection of RSM for storage and distribution within the program. NMEA messages GGA (geographic lat, lon \& alt), GSA (SVs used) and VTG (velocity) are used to create the position record. GSV (SVs in view) is used to create the measurement record. ALM is used to create the GPS almanac record.

\section{CONTROLS}

The RSM can be controlled via three methods: the keyboard, a setup file, and remotely. The keyboard control provides access to parametric controls and program operation through several levels of menus. The setup file controls the initial or default operation of the program when it is started. Remote control is accomplished through a serial interface, and the data formats specified in the RTCM SC104 RSIM message standard [2].

\section{Engineering Display}

The engineering display provides a comprehensive view of all RTCM messages. The components of all received RTCM formatted messages are individually displayed, along with reception time and message ages. In addition to the received pseudorange corrections (PRC's), the display contains the actual PRC values with the range rate correction (RRC) applied as time 


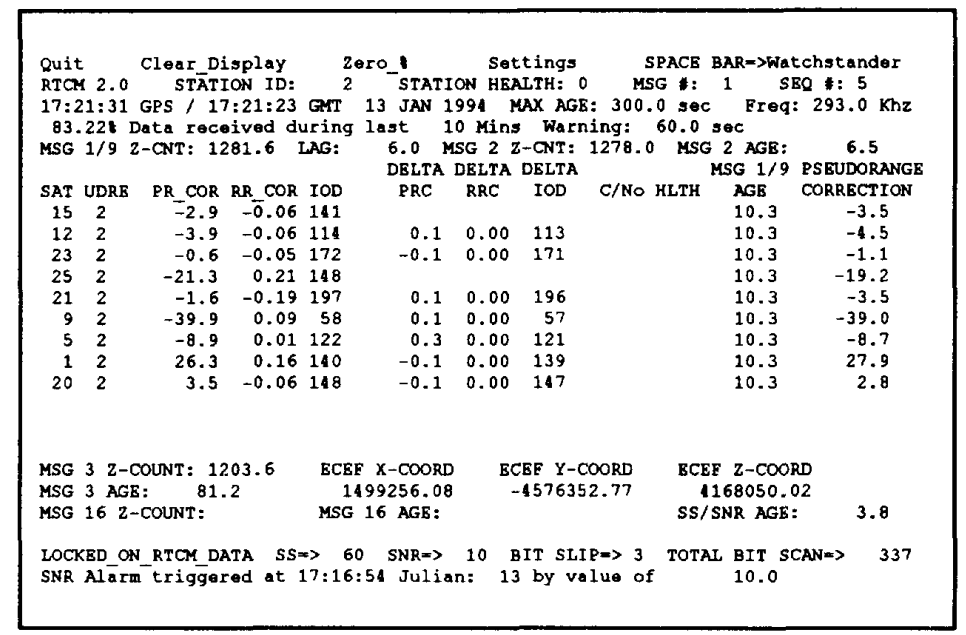

Figure 3 Engineering Display

progresses. All alarms and any system error messages are also displayed. Message Error Rate statistics and radio beacon information are displayed as well. See Figure 3 for a representation of the display.

\section{Watchstander Display}

The watchstander display provides a concise view of alarm criteria and associated measurements. All alarms, and any system error messages are displayed. All of the alarm threshold values can be changed through selection of the "Settings" option on this screen. See Figure 4 for a representation of the display. validation process checks the message checksum first, and then verifies that the contents of each field are within the specified range for that message type. If a problem is found during any part of the validation process, the entire RSIM message is rejected, and a RSIM \#2 message is generated and sent to the Control Station. If the message passes the validation process, an action appropriate to the message type is initiated. Depending on the message type, the actions taken consist of the following possibilities. Configure any or all of the alarm thresholds and/or observation intervals. Respond to queries for a single RSIM message response. Schedule one or more messages for continuous output at specified interval. RSM will only recieve RSIM message types \#1, \#14, and \#16. Any other RSIM message received will generate a RSIM \#2 response.

Alarms: The primary purpose of RSM is to monitor the DGPS broadcast and its contents for system problems or GPS/DGPS anomalies. The alarm capability is utilized to quickly inform the Control Station of any serious problems that may jeopardize user safety and/or the DGPS service operation itself. The RSIM Alarms subprogram contains an independent Ada Task which generates alarm messages. Alarm messages are only generated when the alarm parameter changes state: either when the alarm threshold is exceeded or when the alarm parameter comes back within the acceptable threshold. When triggered, the alarm is generated and sent to the Control Station in the form of a RSIM \#17.

\section{RSIM Manager}

The RSIM Control Package manages all interaction between RSM and RSIM messages and associated actions. The RSIM standard [2], defined by RTCM SC104 , is the prerequisite method for communication to a DGPS integrity monitor. This package is comprised of an independent Ada Task, and several supporting subprograms. The major aspects of RSIM management are RSIM Input, Alarms, Alerts (An internal alarm mechanism placed in RSIM diagnostic message \#5), and RSIM Output.

RSIM Input: The RSIM Input subprogram handles all RSIM messages received by RSM. Each RSIM message received passes through a validation process. The

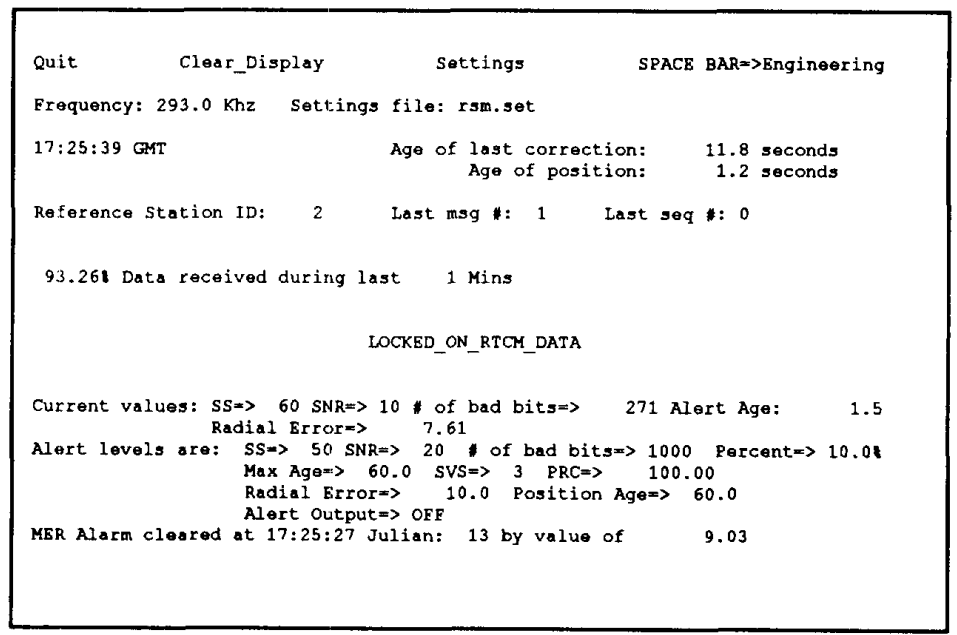

Figure 4 Watchstander Display 
There are eight different alarms, seven of them have two or more possible states or values. Alarms 3-7 use an observation threshold to trigger the actual alarm. The value must exceed the given threshold for the specified observation period. Similarly, once an alarm has been generated, it can only be cleared when the value falls within the threshold for the same specified observation time period. The observation interval values must be carefully chosen by the system providers in order to both prevent nuisance alarms, and minimize notification of real fault conditions. Each of the alarms are discussed below.

1. RTCM Correction Age Alarm. This is also called the Instantaneous Correction Age (ICA) alarm. There are two possible states, ' $H$ ' indicating a high RTCM correction age, and ' $A$ ' indicating an acceptable correction age. The ICA alarm operates in the following manner. Each Pseudo-Range Correction (PRC) age will be checked when received from a RTCM type 1 or 9 message. The age for each PRC will continue to increase, until a new PRC replaces it. An ICA alarm will only be generated when the most recently received PRC age exceeds the threshold value. This means that all PRC ages must exceed the threshold value before the alarm is raised. The alarm will be cleared when a new PRC is received, and its age at time of reception is less then the threshold value. No observation interval is used.

2. High Message Error Ratio Alarm. This referred to as the Message Error Rate (MER) alarm within RSM. There are two possible states, ' $\mathrm{H}$ ' indicating a high value, and ' $A$ ' indicating an acceptable value. The MER alarm is based on the number of properly received RTCM messages, against the number of unusable RTCM messages. The MER value is a percentage, and is determined from a sliding time window of some specified length. The size or length of the window determines how many measurements (data bits) are to be used in the calculation. A large window induces an averaging effect with some latency, while a small window yields more instantaneous results. For MER the observation interval is used to set the sliding window length.

3. Beacon Signal to Noise Ratio (SNR) Alarm. The SNR alarm has two possible states, ' $L$ ' indicating a low SNR value, and ' $A$ ' indicating an acceptable SNR value. This alarm is based on SNR measurements from the MSK Radio Beacon receiver.

4. Beacon Signal Strength (SS) Alarm. The SS alarm has three possible states, ' $Z$ ' indicating zero beacon signal, ' $L$ ' indicating a low beacon signal, and ' $A$ ' indicating an acceptable signal. This alarm is based on
SS measurements from the MSK Radio Beacon receiver.

5. Number of Satellites Alarm. This alarm has three possible states, ' $Z$ ' indicating zero satellites tracked, ' $L$ ' indicating a low number of satellites tracked), and ' $\mathrm{N}$ ' indicating a normal number of satellites. This alarm is based on the number of satellites tracked.

6. HDOP Alarm. This alarm has two states, ' $\mathrm{H}$ ' indicating a high value, and ' $A$ ' indicating an acceptable value. The alarm is based on the HDOP calculation made by the UE.

7. Position Error Alarm. This alarm has two states, ' $\mathrm{H}$ ' indicating a high $2 \mathrm{D}$ position error, and ' $A$ ' indicating an acceptable position error. The value used to determine if an alarm should be generated is based on the 2 dimensional radial error of the position solution from the known antenna location.

8. Reference Station ID Alarm. This alarm is triggered when the RefSta ID within the RTCM data changes from the previously monitored value.

Alerts: The RSM program monitors many different parameters related to DGPS operation and accuracy. Any problem detected that may jeopardize user safety or the DGPS service itself is immediately brought to the attention of the Control Station through the alarm mechanism. Other problems, not considered as catastrophic, are called alerts. These alerts are often indications of system anomalies that do not necessarily require immediate Control Station intervention. RSM uses message type, RSIM \#5, dedicated to Diagnostic Reports and Diagnostic Alarms to report these alerts to the control station. Three of the alert conditions, high Bits Scanned, high SV PRC, and high Position Age are triggered when they exceed their alert threshold values. These threshold values can be modified only through direct interaction with RSM, or by directly editing the ASCII settings file.

1. "Power Up". This indicates that the RSM software has just been started.

2. "No Settings File Found". This indicates that the RSM software could not locate the default settings configuration file, and could not self-configure properly.

3. "Bits Scanned to Lock $=X X X X X X X X "$. When the program loses synchronization with the RTCM data stream, it sets the number of bits scanned since last lock to zero. As the program tries to reacquire synchronization (lock on to the RTCM data stream), the 
number of bits discarded until success are counted. If this value exceeds the corresponding alert threshold, this alert is generated.

4. "SV \#\# PRC = $X X X X X X X X "$. The absolute value of each PRC received by RSM is checked against a corresponding threshold value. If the threshold is exceeded by a particular PRC, then this alert is generated. This alert may occur for each individual PRC, but will not be repeated for any PRC unless a new $P R C$ is received that also exceeds the threshold.

5. "Position Age $=X X X X X X X X "$. This alert is triggered when the time elapsed since the last DGPS/GPS position update exceeds the associated threshold value.

6. "RCVR NOT CONFIGURED". This state indicates that the MSK Beacon receiver has not been configured. Detection of this state is dependant on values of SS and SNR returned by the beacon receiver as specified in the Magnavox MX50R Beacon receiver manual.

7. "NO_MSK_SIGNAL". This indicates that the MSK Beacon receiver is unable to track or detect any signal at the configured frequency. Detection of this state is dependant on values of SS and SNR returned by the beacon receiver as specified in the Magnavox MX50R Beacon receiver manual.

8. "WAITING_FOR_DATA". This state occurs when there is no data being received on the interface selected for RTCM data.

9. "TRYING TO LOCK". This indicates that the RTCM decode process is receiving data, but has not yet found a message preamble, or decoded any RTCM messages.

10. "PREAMBLE DETECTED". This state identifies the point in the decode process when a RTCM preamble has been located, indicating that the beginning of a RTCM message has been found.

11. "LOCKED_ON_RTCM_DATA". This is the ideal or normal state. This indicates that the decode process is locked on to the synchronous RTCM data stream, and is continually decoding valid RTCM messages.

RSIM Output: The RSIM Output subprogram handles any RSIM messages that have been scheduled by RSIM Input to be sent on a continuous basis at some specified interval. The possible RSIM message types are \#14, $\# 15,16, \# 17$, \#18, and \#23. RSIM message types \#15 and \#18 can also echoed to the Data Logger.

RSIM Message Display: The RSIM message display provides a real-time view of all RSIM messages sent or received by RSM in a chronological order. Incoming RSIM messages are displayed in inverse video (highlighted), and outgoing RSIM messages are displayed as normal text. Each message line contains a time of reception or transmission. The display can hold a maximum of twenty inbound and/or outbound RSIM messages. Though this display was originally developed for diagnostic purposes during code development, it has proved to be useful for quick checks of system operation and communications with the Control Station.

\section{CONCLUSION}

With the delivery of RSM version 2.50 in December 1993 the RSM project is on schedule. The final release, version 3.0, is scheduled for July 1994. Version 2.50 gives the Coast Guard a tool to begin conducting evaluations of DGPS service operational methods, test DGPS service control center software and test prospective commercially developed integrity monitors. Upon installation of the DGPS service the RSM tool can then be used as a coverage monitor to validate the range and quality of the DGPS service in specific geographical areas. The design and implementation of RSM makes it maintainable and extensible. New applications such as DGPS/GPS receiver testing, testing of other navigation equipment or more detailed real-time performance analysis of GPS and other radio aid systems could be accomplished by reusing much of the RSM developments.

\section{REFERENCES}

1. 1992 Federal Radionavigation Plan, Department of Transportation, Wash, DC, January 1993.

\section{RTCM Recommended Standards For Differential Navstar/GPS Maritime Reference Stations and Integrity Monitors, Radio Technical Commision for Maritime Services, Wash, DC, December 29, 1993}

\section{RTCM Recommended Standard for Differential} Navstar/GPS Service, version 2.0, Radio Technical Commission for Maritime Services, Wash, DC, January 1990.

4. NMEA 0183 Standard for Interfacing Marine Electronic Devices, Version 2.0, National Marine Electronics Association, Mobile, AL, January 1992 